Article

\title{
Stachys sieboldii Extract Supplementation Attenuates Memory Deficits by Modulating BDNF-CREB and Its Downstream Molecules, in Animal Models of Memory Impairment
}

\author{
Vijaya Abinaya Ravichandran ${ }^{1}$ (1), Mina Kim ${ }^{2}$ (D), Seong Kyu Han ${ }^{3}$ and Youn Soo Cha ${ }^{1, *}$ \\ 1 Department of Food Science and Human Nutrition, Chonbuk National University, 664-14 Duckjin-dong, \\ Jeonju, Jeonbuk 561-756, Korea; vijayaabinaya07@gmail.com \\ 2 Division of Functional Food and Nutrition, Department of Agrofood Resources, National Institute of \\ Agricultural Science, Rural Development Administration, Wanju 55365, Korea; lucidminakim@gmail.com \\ 3 Department of Oral Physiology, School of Dentistry and Institute of Oral Bioscience, Chonbuk National \\ University, Jeonju 561-756, Korea; skhan@jbnu.ac.kr \\ * Correspondence: cha8@jbnu.ac.kr; Tel.: +82-63-270-3822; Fax: +82-63-270-3854
}

Received: 8 May 2018; Accepted: 16 July 2018; Published: 17 July 2018

\begin{abstract}
Cholinergic dysfunction, impaired brain-derived neurotrophic factor and cAMP response element binding protein (BDNF-CREB) signaling are one of the major pathological hallmarks of cognitive impairment. Therefore, improving cholinergic neurotransmission, and regulating the BDNF-CREB pathway by downregulating apoptosis genes is one strategy for inhibiting the etiology of dementia. This study evaluates the potential effects of Stachys sieboldii MIQ (SS) extract against cognitive dysfunction and its underlying mechanisms. SS supplementation for 33 days improved scopolamine-induced memory impairment symptoms in Morris water maze test and Y-maze test. SS reduced the acetylcholineesterase activity and significantly increase acetylcholine and cholineacetyltransferase activity in the brain. In the subsequent mechanism study, SS regulated the mRNA expression level of neuronal plasticity molecules such as (nerve growth factor) NGF, BDNF, CREB, and its downstream molecules such as Bcl-2 and Egr-1 by downregulating the neuronal apoptosis targets in both hippocampus and frontal cortex. Additionally, inward currents caused by SS in hippocampal CA1 neurons was partially blocked by the GABA receptor antagonist picrotoxin $(50 \mu \mathrm{M})$, suggesting that SS acts on synaptic/extrasynaptic $\mathrm{GABA}_{\mathrm{A}}$ receptors. These findings indicate that SS may function in a way that is similar to nootropic drugs by inhibiting cholinergic abnormalities, and neuronal apoptosis targets and ultimately increasing the expression of BDNF-CREB.
\end{abstract}

Keywords: Stachys sieboldii; dementia; memory loss; cholinergic neurotransmission; neuroplasticity targets; $\mathrm{GABA}_{\mathrm{A}}$ receptor

\section{Introduction}

Dementia is a current epidemic (approx. 46.8 million new cases worldwide in 2015) and public health and economic burden that attracts increasing investment into research [1]. The disease is marked by a slow, progressive, and irreversible decline in neurocognitive functioning over the years that affects memory, language, and problem-solving abilities [2]. Accumulating evidence and post-mortem studies of the brains of patients with dementia revealed low levels of the acetylcholine (ACh) and choline acetyltransferase (CAT) and increased level of acetylcholinesterase (AChE) [3,4]. Therefore, cholinergic abnormalities have received specific attention and most therapies for dementia are directed to this system [5]. 
Numerous molecules related to cognitive function have been identified recently. Among them, the first discovered neurotrophin, nerve growth factor (NGF), regulates biological mechanisms related to neuronal plasticity in the brain [6]. The second discovered neurotrophin is brain-derived neurotrophic factor (BDNF), and its major receptor tropomyosin receptor kinase B (TrkB) are involved in synaptic plasticity and memory processes in both the hippocampus and cortical neurons [7]. BDNF is also involved in long term memory (LTM) formation in the CA1 region of the hippocampus, via the activation of the transcription factor cAMP response element binding protein (CREB) [8]. CREB leads to neuronal survival through the regulation of downstream target genes such as B-cell lymphoma 2 ( $B c l 2)$ [9] and mediates neuronal plasticity by early growth response 1 (EGR1) [10]. Conversely, neuronal apoptosis carried out by two main pathways, one of which is the intrinsic pathway driven by Bax leading to mitochondrial outer membrane permeabilization (MOMP) [11]. The increase in MOMP results in release of cyclooxygenase-2 (COX-2) into the cytoplasm [12]. This, in turn, binds to caspase-9 (extrinsic pathway activates), which, recruits and activates the executioner caspase-3 leading to neuronal cell death [13]. These neuronal metabolic pathways demonstrate the possibility that dementia could be ameliorated or prevented by activation of neurotrophic factors or/and by inhibition of neuronal apoptosis.

$\gamma$-aminobutyric acid (GABA) begins as the key excitatory neurotransmitter in newly forming circuits, with chloride efflux from GABA type A receptors $\left(G_{A B A} R s\right)$ producing membrane depolarization, dendritic outgrowth, and synaptogenesis [14]. GABA $A$ R activation also controls the formation and plasticity of GABAergic synapses, results in the normalizing the cognitive impairment [15]. In addition, pharmacological findings show that traditional drugs acting at GABAergic receptors have memory active properties [16,17].

Many synthetic drugs have been introduced for the treatment of dementia and ultimately withdrawn from the market due to severe toxicity and side effects. Therefore, alternative approaches, such as the use of natural products for the development of functional foods with neuroprotective effects, have been of great research interest [18]. The genus Stachys (Lamiaceae) includes about 200-300 species in the world. Stachys sieboldii is erect, hairy, perennial, stoloniferous herb, which is indigenous to China; and in Japan and Korea it has been cultivated since ancient times [19]. The extracts of the plant have been used as folk medicine against several infections for many decades. Phytochemical investigation of Stachys sieboldii have shown the occurrence of flavonoids, diterpenes, phenyl ethanoid glycosides and saponins [20]. They are also a good source of oligosaccharides, proteins, and water-soluble vitamins (vitamin B complex), which are all major contributors to human nutrition [21]. Several reports have detailed the health benefits of Stachys sieboldii, such as its anti-inflammatory activity, ability to lower anoxia, immunosuppressive function, antinephritic activity, antioxidant, antimicrobial activity, they also improve memory and discomfort [20-23].

In agreement, we have previously reported protective effects of Stachys sieboldii MIQ (SS) against $\mathrm{H}_{2} \mathrm{O}_{2}$ induced cytotoxicity in SK-N-SH cells (Human neuroblastoma cell line) and memory amelioration in mice [22]. Notably, an underlying molecular mechanism by which SS exert the neuroprotective effect has not yet been studied. Therefore, the aim of the present investigation was to elucidate the underlying mechanisms by which SS ameliorates learning and memory ability in a scopolamine-induced amnesia animal model with particular emphasis on cholinergic neurotransmission. Moreover, whole cell patch clamp assays were also used to examine the direct membrane effect of the SS as well as its effects on GABA currents in hippocampal CA1 neuron.

\section{Materials and Methods}

\subsection{Preparation of Stachys Sieboldii Extract}

Stachys sieboldii was supplied by the Farmer's morning Wholesaler, (Gochang, Jeonbuk, South Korea) and was washed. Then SS were freeze dried at $-40{ }^{\circ} \mathrm{C}$ for $48 \mathrm{~h}$, and extracted with $20 \%$ ethanol at $40{ }^{\circ} \mathrm{C}$ for $4 \mathrm{~h}$. The resultant was filtered, concentrated to $128 \mathrm{brix}$, and again freeze dried at $-40{ }^{\circ} \mathrm{C}$ 
for $48 \mathrm{~h}$. The SS extracts were ground with a 50- $\mu \mathrm{m}$ mesh to yield a powdered sample.UHPLC-MS/MS analysis for crude extract of Stachys Sieboldii have been added in supplementary materials (Table S1 and Figure S1).

\subsection{UHPLC-MS/MS}

UHPLC-MS/MS analyses were performed using a Shimadzu UHPLC (SIL-30A) coupled with a triple quadrupole mass spectrometer Shimadzu ESI-MS (8040). Chromatography was carried out using a Waters ACQUITY UPLC ${ }^{\circledR}$ BEH HILIC $1.7 \mu \mathrm{m}(2.1 \times 100 \mathrm{~mm}$ column $)$ at flow rate of $0.5 \mathrm{~mL} \mathrm{~min}^{-1}$, with an injection volume of $1 \mu \mathrm{L}$. The mobile phase was formed by solvent A ( $5 \mathrm{mM}$ ammonium formate, $0.2 \%$ formic acid in 50\% ACN (acetonitrile)) and solvent B $30 \mathrm{mM}$ ammonium formate, $0.2 \%$ formic acid in 50\% ACN (acetonitrile). The column temperature was set at $40{ }^{\circ} \mathrm{C}$. The following gradient was used: $0 \mathrm{~min}, 100 \% \mathrm{~A} ; 2 \mathrm{~min}, 100 \% \mathrm{~A} ; 25 \mathrm{~min}, 40 \% \mathrm{~B} ; 40 \mathrm{~min}, 100 \% \mathrm{~A} ; 45 \mathrm{~min}, 100 \% \mathrm{~A}$. Electrospray ionization (ESI) source, operated in the positive mode and quantitated using selected reaction monitoring (SRM) transitions of $\mathrm{m} / \mathrm{z} 104.1-60.2,45.3$ (Figure 1).

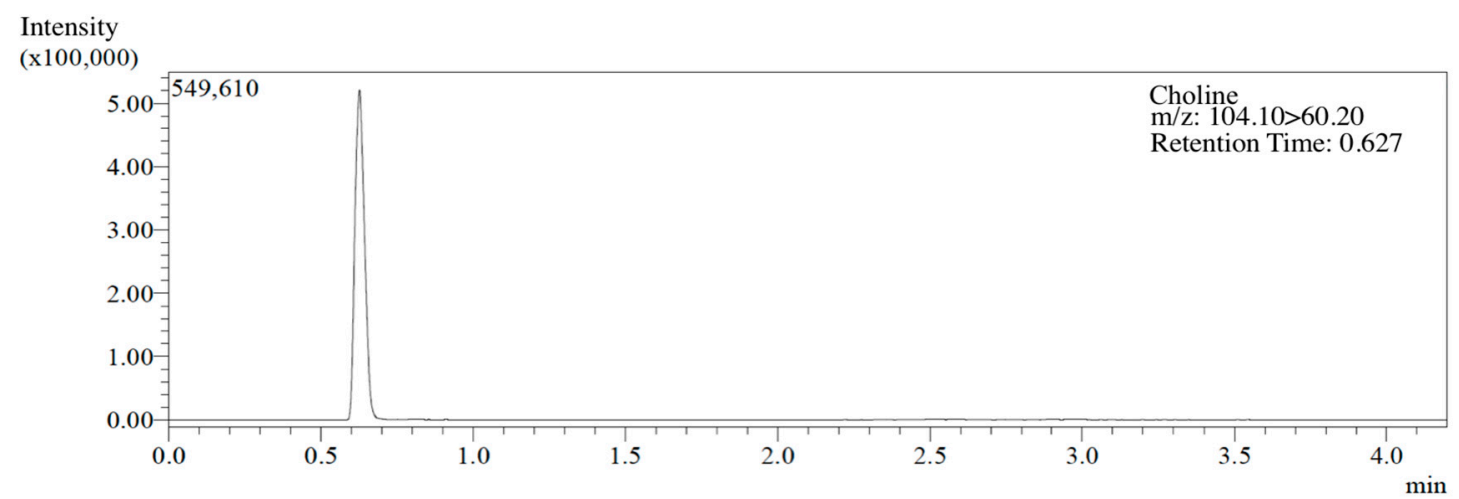

Figure 1. UPLC/MS/MS chromatogram of a Stachys sieboldii MIQ extract containing choline.

\subsection{Animals and Experimental Groups}

All animal procedures were approved by the Animal Care and Use Committee of Chonbuk National University (CBNU 2016-42). Male Sprague-Dawley rats and ICR mice were purchased at 5 weeks of age (Charles River Laboratory, Tokyo, Japan). The animals were housed in individual cages with free access to water and commercial AIN-76A diet (Research Diets, New Brunswick, NJ, USA) in a room with a $12 \mathrm{~h} / 12 \mathrm{~h}$ light-dark cycle, at temperature of $23 \pm 1{ }^{\circ} \mathrm{C}$, and humidity of $50 \pm 5 \%$. After a one-week adaptation period, animals were randomly divided into four groups ( $n=10$ per group): (1) control group (C) (vehicle intraperitoneal (i.p.) + vehicle per os (p.o.)); (2) Scopolamine group (Scop) (scopolamine i.p. + vehicle p.o.) (Sigma Aldrich, St. Louis, MO, USA); (3) Donepezil group (D + Scop) (positive control) [24] (Abcam, Cambridge, UK) (scopolamine i.p. + Donepezil $5 \mathrm{mg} / \mathrm{kg}$ body weight p.o.); and (4) SS group (SS + Scop) (scopolamine i.p. + SS 500 and/or $250 \mathrm{mg} / \mathrm{kg}$ body weight p.o.). Morris water maze study was conducted in rats with SS concentration of $250 \mathrm{mg} / \mathrm{kg}$ body weight p.o. which is equal to $500 \mathrm{mg} / \mathrm{kg}$ body weight p.o. in mice according to the equivalent surface area dosage conversion factors [25]. Both donepezil and the SS extract were dissolved in distilled water immediately before use and orally administered to animals for continuous 28 days (pretreatment) at a dose of 5250 , or $500 \mathrm{mg} / \mathrm{kg}$ body weight, respectively for both Morris water maze and Y-maze experiments. Memory impairment was induced by scopolamine (SCO) treatment $(1 \mathrm{mg} / \mathrm{kg}$ body weight, i.p.) $30 \mathrm{~min}$ before each task. In the control group, the vehicle solution (saline, i.p.) was administered using the same time schedule (Figure 2A). The $200 \mathrm{mg}$ of SS extract contains $0.0676 \mathrm{mg} / \mathrm{g}$ choline concentration of dry weight. 


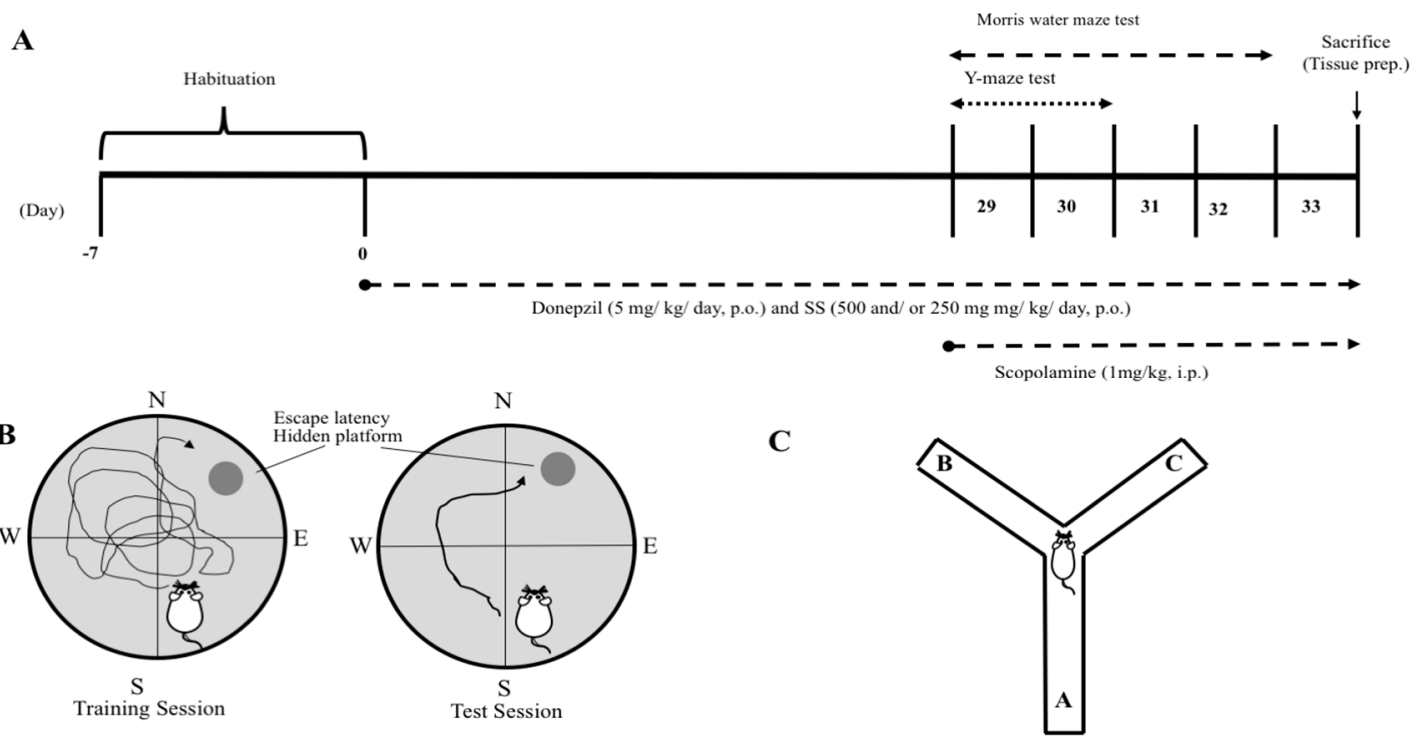

Figure 2. Animal experimental procedure. Schematic representation of the animal experimental protocol (A). Memory assessment paradigms used in this study Morris water maze (B) and Y-maze (C).

\subsection{Morris Water Maze Test}

The Morris water maze task was performed based on the paradigm of Morris, 1985 with slight modifications. (Figure 2B) [26]. The experimental apparatus consisted of a circular pool (diameter, $100 \mathrm{~cm}$; height, $135 \mathrm{~cm}$ ) containing water at $22 \pm 1^{\circ} \mathrm{C}$ that was rendered opaque by the addition of powdered milk. The pool was equally divided into quadrants. A platform was positioned inside the tank with its top submerged $1 \mathrm{~cm}$ below the water surface in the target quadrant of the maze. In our experiment we have assessed the acquisition in terms of latency to locate the escape platform. The test is based on two phases; the acquisition phase (Training days) and the retention phase (Probe trial). Initially, the training session was performed during which each rat was placed into the water facing toward the wall of the tank. After placing, $300 \mathrm{~s}$ were given to each animal to find and mount onto the hidden platform. If it failed to locate the platform during the allocated time, then it was guided gently to the platform and allowed to stay on it for $30 \mathrm{~s}$. Each rat received one training sessions and was repeated 2 times at $10 \mathrm{~min}$ intervals for three consecutive days in acquisition training. The probe trial was performed on 4 th day after the training phase. The video was recorded using the video tracking system (Ethovision System, Noldus, Wageningen, The Netherlands). Thirty minutes before experiment rats were intraperitoneally injected with scopolamine $(1 \mathrm{mg} / \mathrm{kg}$, i.p.).

\subsection{Y-maze Test}

The Y-maze is a three-arm horizontal maze with an angle of 120 degrees, which were $28 \mathrm{~cm}$ length, $6 \mathrm{~cm}$ width, and $18 \mathrm{~cm}$ height. The maze floor and walls were constructed with white polyvinyl plastic. Mice were initially placed in one arm, and then the sequence and number of arm entries were monitored for an 8-min period. An actual alternation was defined when a mouse entered into all three arms on consecutive choices (i.e., $\mathrm{ABC}, \mathrm{BCA}$, or $\mathrm{CAB}$, but not $\mathrm{CAC}, \mathrm{BAB}$, or $\mathrm{ABA}$ ). The spontaneous alternation (\%) was derived from the total number of alternations divided by the total number of arm entries minus two, which was multiplied by 100 as shown in the following equation: $\%$ Alternation $=$ [(Number of alternations)/(Total number of arm entries -2$)] \times 100$. The number of arm entries also served as an indicator for movement and locomotor activity (Figure 2C) [27]. 


\subsection{Sample Collection}

At the end of the behavioral test, animals were decapitated immediately, to collect brains. All brain samples were careful excised, rinsed, and immediately stored at $-70^{\circ} \mathrm{C}$ until analysis was done.

2.7. Determination of Acetylcholine, Choline Acetyl Transferase, and Acetyl Choline Esterase Levels in Animal Model of Impaired Memory

ACh, AChE, and CAT levels were analyzed in the hippocampus and cortex of the collected brain sample. The hippocampus and cortex were separated, rinsed in cold PBS, resuspended in PBS, and homogenized. The homogenates were centrifuged at $1500 \times \mathrm{g}$ for $15 \mathrm{~min}$ at $4{ }^{\circ} \mathrm{C}$. The supernatants were used in an ACh, AChE, and CAT assay using commercially available kits (MyBioSource, San Diego, CA, USA).

\subsection{Quantitative Reverse Transcription PCR}

Total RNA was isolated from hippocampus and cortex using Trizol reagent (Life Technologies, Inc., Carlsbad, CA, USA). The concentration and purity of RNA was measured spectrophotometrically on a Biodrop Duo instrument (Biochrom, Holliston, MA, USA) calculating the 260:280 absorbance ratio. The extracted RNA was reverse transcribed into cDNA using a high capacity cDNA reverse transcription kit (Applied Biosystems, Foster City, CA, USA). RNA expression levels were quantified using SYBR Green real-time PCR master mix (TOYOBO, Osaka, Japan). Thermocycling was performed on a 7500 Real Time PCR system (Applied Biosystems). Primers used are given in Table 1.

Table 1. Primer sequence used in the animal study.

\begin{tabular}{ccc}
\hline Gene Name & Primers & Sequence $\left.\mathbf{( 5}^{\prime}-\mathbf{3}^{\prime}\right)$ \\
\hline \multirow{2}{*}{ NGF } & Forward & ACCTCTTCGGACACTCTGG \\
& Reverse & CGTGGCTGTGGTCTTATCTC \\
\hline \multirow{2}{*}{ BDNF } & Forward & CGAGACCAAGTGTAATCCCA \\
& Reverse & TCTATCCTTATGAACCGCCA \\
\hline \multirow{2}{*}{ Trkb } & Forward & TCTCATTTTAGGCCGCTTTG \\
& Reverse & GGGTTTGAGGTGGGTGAAG \\
\hline \multirow{2}{*}{ CREB } & Forward & TACCCAGGGAGGAGCAATAC \\
& Reverse & GAGGCAGCTTGAACAACAAC \\
\hline \multirow{2}{*}{ Egr-1 } & Forward & CCAGTGCCCACCTCTTACTC \\
& Reverse & TGCAGACTGGAAGGTGCTG \\
\hline \multirow{2}{*}{ Bcl-2 } & Forward & TTGACGCTCTCCACACACATG \\
& Reverse & GGTGGAGGAACTCTTCAGGGA \\
\hline \multirow{2}{*}{ Bax } & Forward & CTGGAAGAAGATGGGCTGAGG \\
\multirow{2}{*}{ Cox-2 } & Reverse & ACCTGAGGTTTATTGGCACCT \\
\hline \multirow{2}{*}{ Caspase-3 } & Forward & GGCACAAATATGATGTTCGC \\
& Reverse & CCTCGCTTCTGATCTGTCTTGA \\
\hline \multirow{2}{*}{ GAPDH } & Forward & AATTCAAGGGACGGGTCATG \\
& Reverse & GCTTGTGCGCGTACAGTTTC \\
\hline \multirow{2}{*}{ Fospard } & Forward & CTGTCCCGTGAAGCAAGGAT \\
& Reverse & TGGTACATCGGCAGAGAAGC \\
\hline
\end{tabular}

\subsection{Brain Slice Preparation and Electrophysiology}

Brain slices were prepared as described by [17] earlier study with slight modifications in position of brain used. Immature ICR male mice (5-20 Postnatal days) were sacrificed by cervical dislocation 
between 10:00 and 12:00 $\mathrm{h}$ and their brains rapidly removed and placed in the ice-cold low calcium $(0.5 \mathrm{mM})$, high magnesium $(6 \mathrm{mM})$ bicarbonate-buffered artificial cerebrospinal fluid (ACSF) ( $\mathrm{pH} 7.4$ when bubbled with $95 \% \mathrm{O}_{2}$ and $\left.5 \% \mathrm{CO}_{2}\right)$ ). Brains were then cut into 150 to $200 \mu \mathrm{m}$-thick coronal slices using a vibratome (Microm, Walldorf, Germany). Patch pipettes (4-6 M $\Omega$ ) were pulled from thin-wall borosilicate glass-capillary tubing (outer diameter, $1.5 \mathrm{~mm}$; inner diameter, $1.17 \mathrm{~mm}$ ) (PG52151-4, WPI, Sarasota, FL USA) on a horizontal Flaming/Brown puller (P-97; Sutter Instruments Co., Novato, CA, USA). The pipette solution was passed through a disposable $0.22 \mu \mathrm{m}$ filter [28]. The whole cell patch clamp recordings were performed under voltage clamp using an Axopatch 200B (Axon Instruments, Union City, CA, USA). The cells were voltage clamped at- $60 \mathrm{mV}$ after nullifying the junction potential between the patch pipette and bath solution. The changes in membrane current were sampled online using a Digidata 1322A interface (Axon Instruments, Union City, CA, USA). Acquisition and subsequent analysis of the acquired data were performed using Clampex 9 software (Axon Instruments, USA). All recordings were made at room temperature.

\subsection{Statistical Analysis}

Behavioral studies were analyzed by one-way ANOVA using SPSS version 17.0 (SPSS Institute, Chicago, IL, USA). Values are expressed as means \pm standard deviations. The significance of differences between groups was assessed using Tukey's test. Electrophysiological studies values are expressed as the mean \pm S.E.M. One-way ANOVA was performed to analyze more than two experimental groups using origin 7.0. Student's $t$-test was used to examine the differences between the two experimental groups. $p<0.05$ was considered significant.

\section{Results}

\subsection{Effects of SS on Memory Function in Animal Model of Impaired Memory}

The dose of choline that was administered to the animals was $0.169 \mathrm{mg} /$ day [25]. The effects of SS administration following scopolamine induced memory impairments in animals are shown in Figures 3 and 4 . The Control group showed the ability to learn normally, as seen by a significantly decreased in escape latency of day 3 compared to day 1 and day 2 compared to scopolamine treated group $(p<0.05)$ (Figure 3A). However, pretreatment with SS prevented the impairment effects of scopolamine on memory as evidenced by a significant decrease in escape latency time (day 3 ) in the SS + Scop group compared to the scopolamine alone treated group $(p<0.05)$. We further investigated swimming speed (i.e., velocity) by dividing the escape path length by the escape latency period (Figure 3B). A significant increase in velocity was observed in scopolamine group compared to other groups in all three days. The probe trial was performed $24 \mathrm{~h}$ after the last training session (Figure 3C,D). Compared to scopolamine treated group, animals treated with SS and donepezil remained longer time in the target quadrant $(p<0.05)$. The SS + Scop group also showed a significantly decreased velocity (two-fold) compared to the animals treated with scopolamine alone. These results indicate that SS improved spatial memory.

In a Y-maze test, a higher alternation rate demonstrates more sustained cognition, as the animals do not reenter the same arm. Scopolamine group exhibited a significantly decreased spontaneous alternation percentage $(40.74 \% \pm 3.52 \%)$ compared with the control group $(61.6 \% \pm 9 \%)(p<0.05)$. Nevertheless, we observed a remarkable increase in working memory in the SS + Scop treated group $(p<0.05)$, indicated by an increase in spontaneous alternation percentage, compared to the scopolamine-treated group (Figure 4A). Additionally, the number of arm entries was recorded and used as an indicator of locomotor activity. As shown in Figure 4B, scopolamine group had a significantly lower number of arm entries compared to the control group; however, animals treated with donepezil and the SS showed increased number of arm entries compare to scopolamine treated group $(p<0.05)$. 


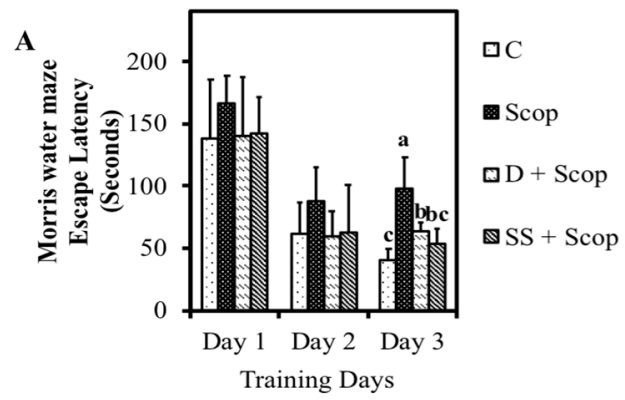

B
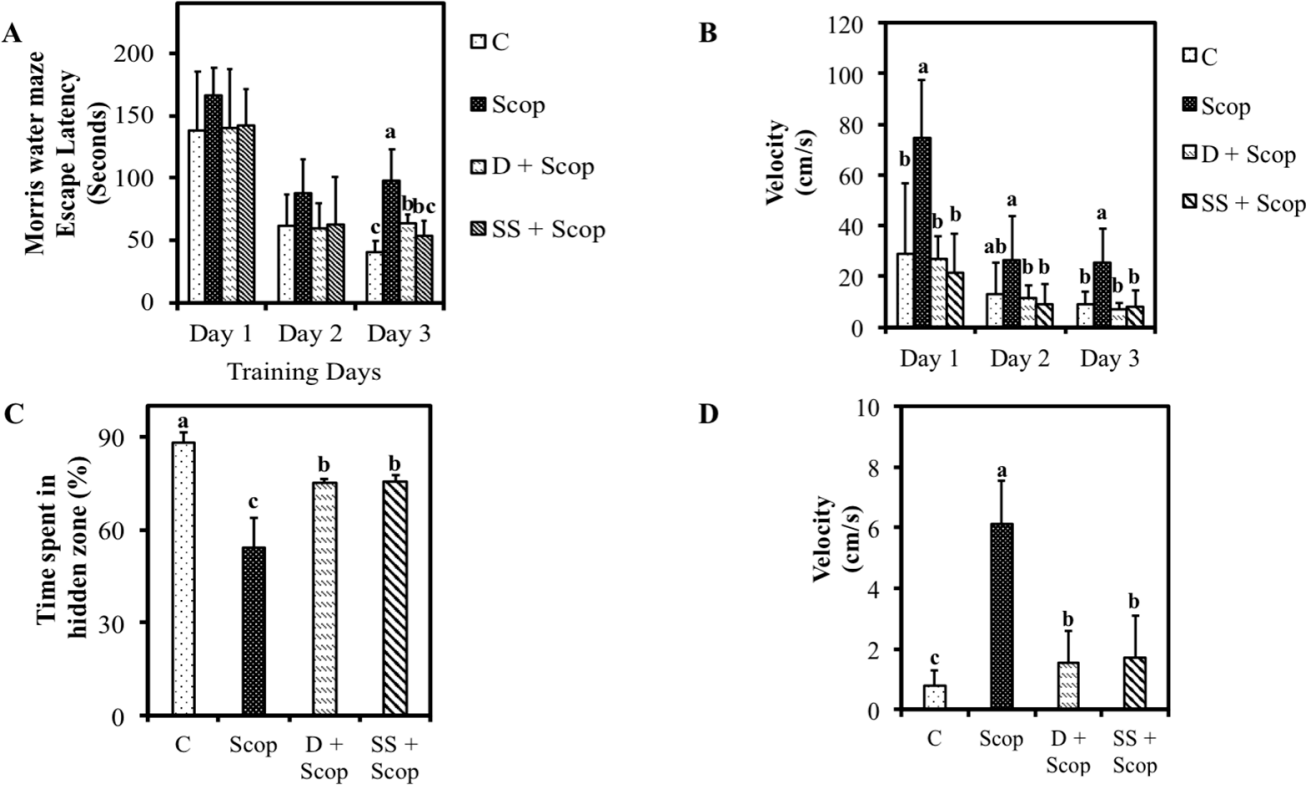

D

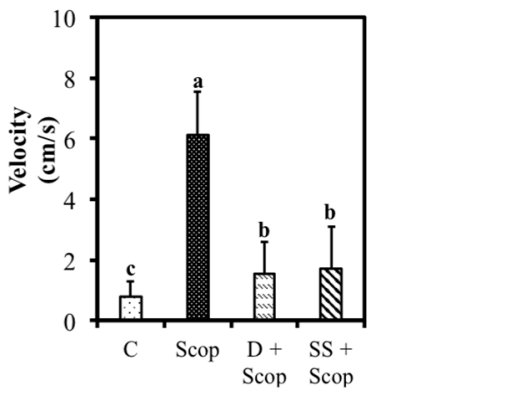

Figure 3. Effect of Stachys sieboldii in scopolamine induced memory impairment was assessed by Morris water maze. SS reduced escape latency (A), increased velocity (B), time spent in hidden zone during probe trial (C) and velocity (D) All values are mean \pm SD $(n=10)$. Mean with different superscripts in the same row indicates significant difference by ANOVA with Tukey's test at $p<0.05$ compared to control group.

A

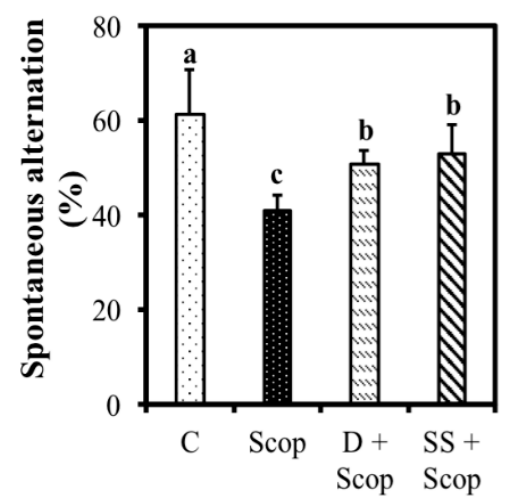

B

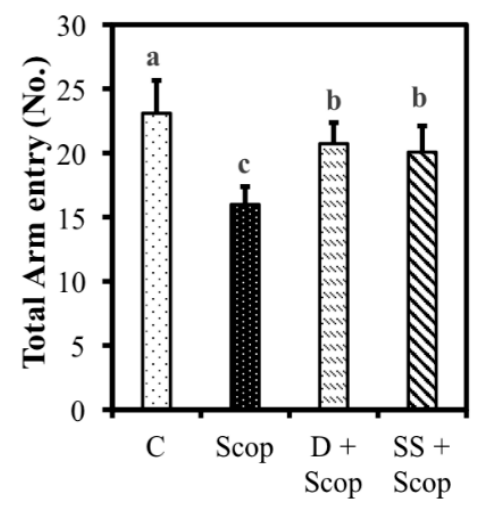

Figure 4. Effect of Stachys sieboldii in scopolamine induced memory impairment was assessed by Y-maze. SS reduced spontaneous alternation (\%) (A), and Total arm entry (No.) (B). All values are mean $\pm \mathrm{SD}(n=10)$. Mean with different superscripts in the same row indicates significant difference by ANOVA with Tukey's test at $p<0.05$ compared to control group.

\subsection{Effects of SS on Hippocampal and Frontal Cortical, ACh, CAT, and AChE Levels in Animal Model of Impaired Memory}

The levels of ACh, CAT, and AChE in the hippocampus and frontal cortex are presented in Figure 5. Compared with the control group, the scopolamine group exhibited decreased level of ACh in both the hippocampus and cortex $(p<0.05)$. Treatment with either donepezil or SS resulted in a significantly increased level of $\mathrm{ACh}$ in animals compared to animals treated with scopolamine alone (Figure 5A). As shown in Figure 5B, CAT levels in the hippocampus did not differ significantly among the groups; however, CAT level showed increased tendency in the donepezil- and SS-treated animals compared to the scopolamine group. Cortical levels of CAT were increased significantly in the control 
group than in the scopolamine group. Furthermore, in animals treated with either donepezil or SS significantly increased the CAT level in the cortical area compared to scopolamine group $(p<0.05)$. The scopolamine-treated group showed an increased level of AChE compared to the control group in both the hippocampus $(p<0.05)$ and cortex $(p>0.05)$. Interestingly, animals treated with either donepezil or SS showed markedly similar levels of AChE to the control group in both the hippocampus $(p<0.05)$ and cortex $(p>0.05)$ (Figure 5C).

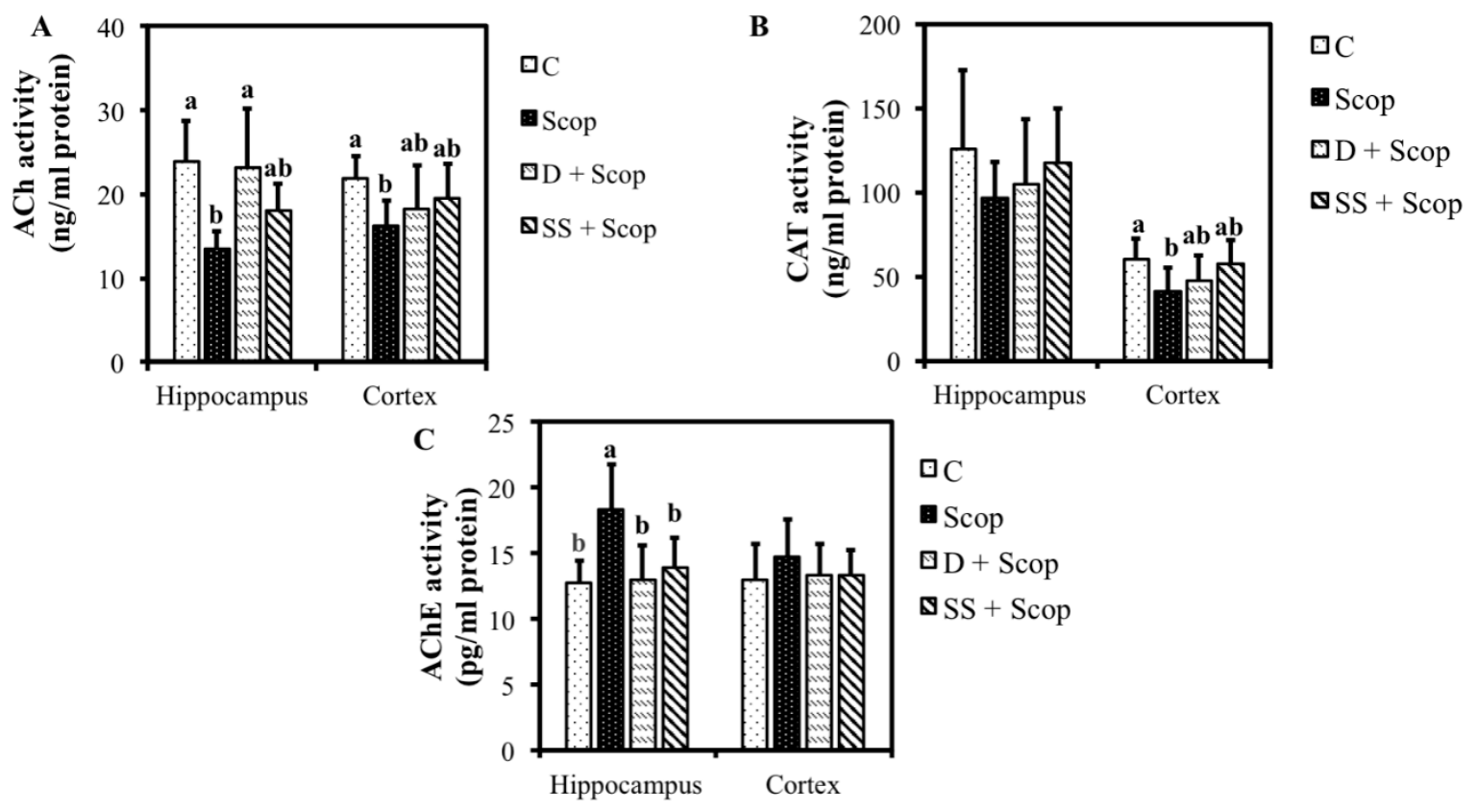

Figure 5. Effect of Stachys sieboldii in hippocampus and frontal cortex of animal brain Acetylcholine (A) Choline acetyl transferase, (B) and Acetylcholine esterase level (C). All values are mean \pm SD $(n=10)$. Mean with different superscripts in the same row such as $\mathrm{a}, \mathrm{b}$ indicates significant difference by ANOVA with Tukey's test at $p<0.05$ compared to control group.

\subsection{Effect of SS on mRNA Expression in the Hippocampal and Frontal Cortical Area on Memory Impaired Animals}

To understand the mechanisms through which SS protects scopolamine-induced memory impairment, the status of several components of the pathway that regulates neuroplasticity in the hippocampus and frontal cortex was examined using mRNA expression. Scopolamine markedly downregulated NGF, BDNF, and Trkb mRNA expression levels in the hippocampus (Figure 6) and frontal cortex (Figure 7). Meanwhile, SS significantly upregulated a scopolamine-induced decrease in NGF, BDNF, Trkb mRNA expression levels. Furthermore, the expression levels of CREB, Egr-1, and $\mathrm{Bcl}-2$ were investigated in the hippocampus and frontal cortex, as these molecules are implicated in learning and memory processes. Treatment with SS restored the downregulation of CREB, Egr-1, and Bcl-2 induced by scopolamine in the hippocampus and frontal cortex. Simultaneously, Bax, COX-2, caspase-3, and caspase-9 are involved in the neuronal apoptosis pathway in the hippocampus (Figure 6 G-H) and frontal cortex (Figure 7G-H). The mRNA expression levels of Bax, COX-2, caspase-3, and caspase- 9 were found to be downregulated in the SS + Scop group compared to the Scopolamine only group. These data demonstrate that the SS attenuates memory-impairment effect induced by scopolamine through activation of NGF-BDNF-CREB signaling in the frontal cortex and hippocampus. 
A

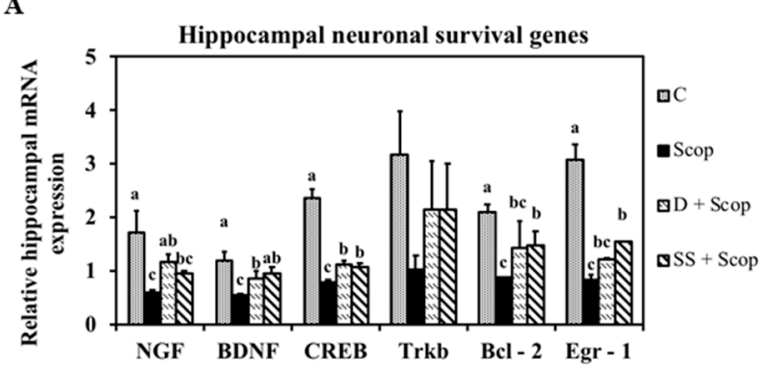

B

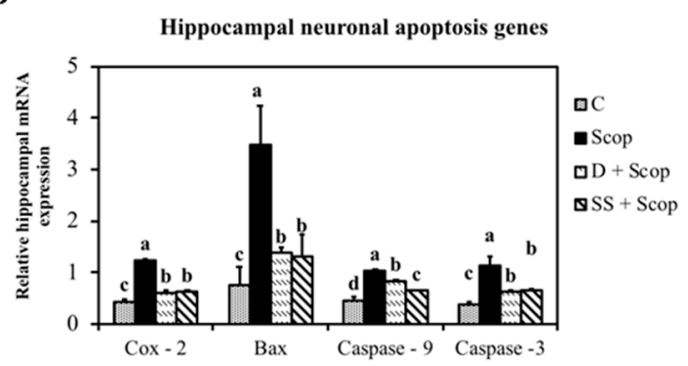

Figure 6. Effect of Stachys sieboldii in the mRNA expression of hippocampal neuronal survival genes (A) and neuronal apoptosis genes $(\mathbf{B})$ All values are mean $\pm \operatorname{SD}(n=4)$. Mean with different superscripts in the same row such as $\mathrm{a}, \mathrm{b}$ indicates significant difference by ANOVA with Tukey's test at $p<0.05$ compared to control group.

$\mathbf{A}$

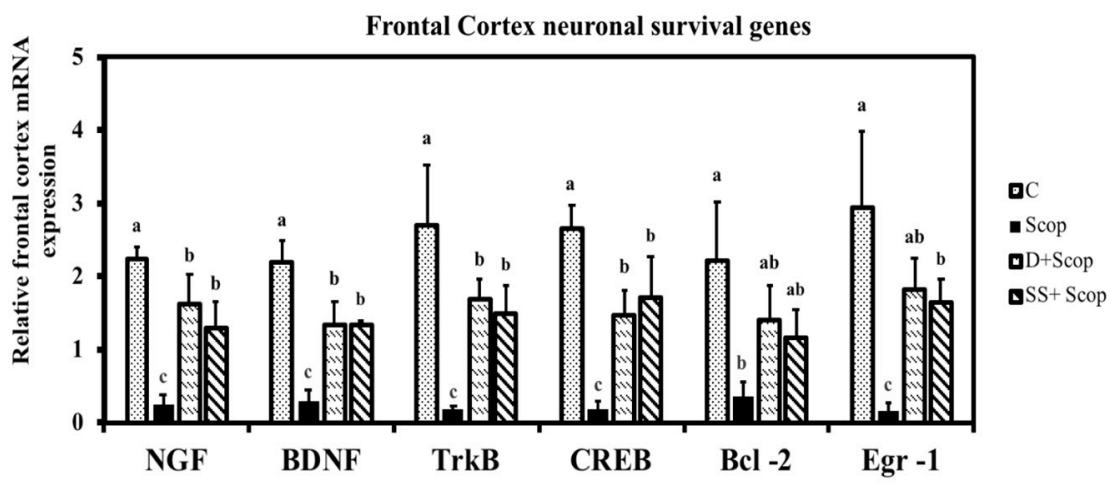

B

Frontal Cortex neuronal apoptosis genes

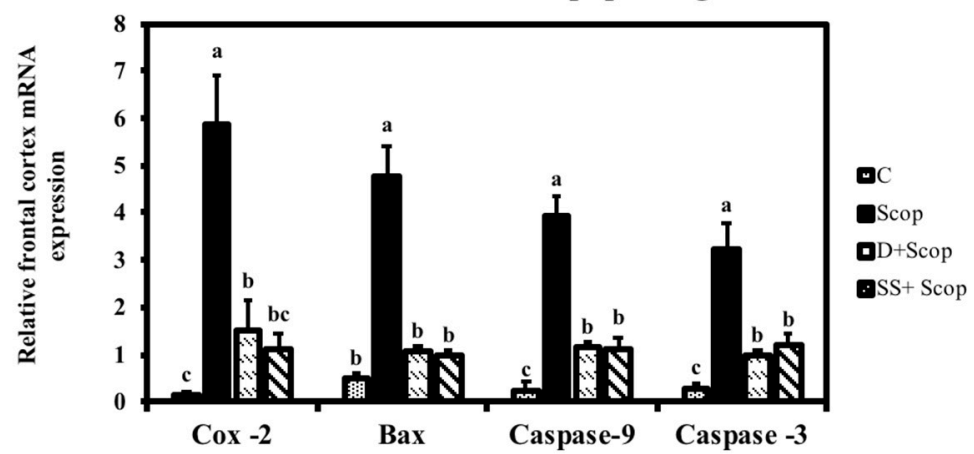

Figure 7. Effect of Stachys sieboldii in the mRNA expression of frontal cortical neuronal survival genes (A) and neuronal apoptosis genes (B) All values are mean $\pm \mathrm{SD}(n=4)$. Mean with different superscripts in the same row such as $\mathrm{a}, \mathrm{b}$ indicates significant difference by ANOVA with Tukey's test at $p<0.05$ compared to control group.

\subsection{Electrophysiology Results}

Whole cell currents were recorded from CA1 pyramidal neurons in the hippocampal region of immature male mice brains. Under whole-cell, voltage clamp, high chloride pipette solution conditions, SS was applied in concentration-dependent manner. Bath application of 1, 3, 10, 30, 100, or $300 \mu \mathrm{g} / \mathrm{mL}$ of SS revealed a clear, concentration-dependent increase in the SS-induced inward currents (Figure $8 \mathrm{~A}, \mathrm{~B})(p<0.05)$. In another set of experiments, inward currents induced by the application of SS $(100 \mu \mathrm{g} / \mathrm{mL})$ were reproducible, and the response induced by the second application 
of SS was similar to that of the first application (Figure $8 \mathrm{C}, \mathrm{D}$ ), proving its desensitization effect on CA1 neurons. The effect of SS on CA1 pyramidal neurons was evaluated in the presence of TTX $(0.5 \mu \mathrm{M})$ to determine if SS acts directly on CA1 pyramidal neurons and not via any action potential-mediated presynaptic release. TTX failed to inhibit the SS-induced inward current in all (5/5) CA1 pyramidal neurons (Figure 8E,F), which shows that SS has a direct effect on CA1 neurons. We also examined whether SS affected the GABA receptors of CA1 pyramidal neurons through successive application of a GABA receptor antagonist. As shown in Figure 8G, the SS-induced inward currents were blocked

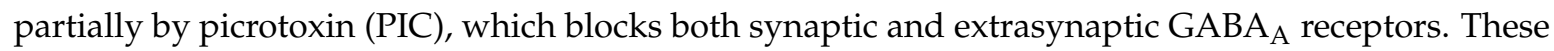
results suggest that $\mathrm{SS}$ acts on both synaptic and extrasynaptic $\mathrm{GABA}_{\mathrm{A}}$ receptors (Figure $8 \mathrm{H}$ ).

A

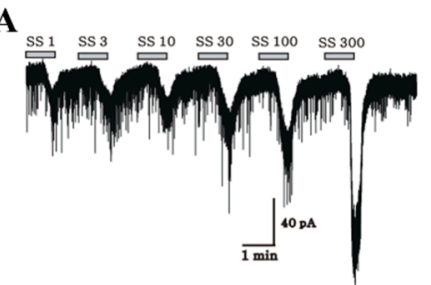

C

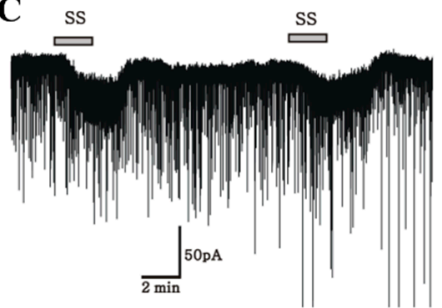

E

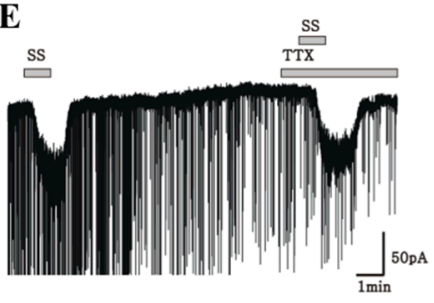

B

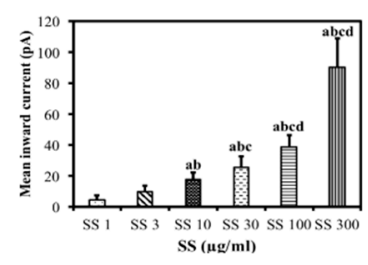

D

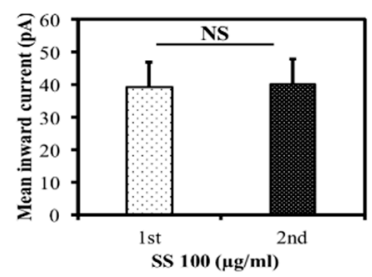

F
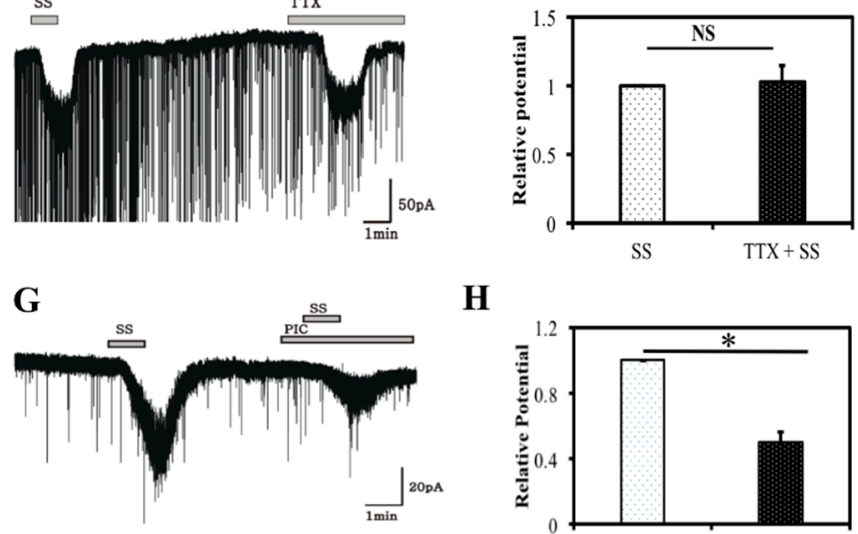

H

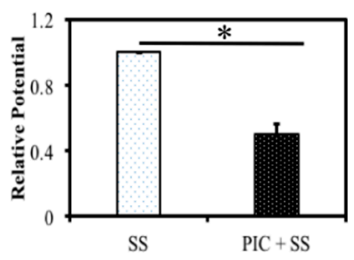

Figure 8. Effect of Stachys sieboldii on juvenile CA1 neuron. Concentration-dependent increase in SS-induced inward currents (A) and bar graph of Figure A (B). Reproducible non-desensitizing effect of SS $(100 \mu \mathrm{g} / \mathrm{mL})$ on a juvenile CA1 neuron (C), Bar graph shows the mean relative inward currents induced by SS $(100 \mu \mathrm{g} / \mathrm{mL})(D)$. The SS-induced inward currents persisted in the presence of TTX $(0.5 \mu \mathrm{M})(\mathbf{E})$. Bar graph shows the mean inward currents by SS (F). The SS-mediated inward currents occurred partly through the extrasynaptic $\mathrm{GABA}_{\mathrm{A}}$ receptors. A current trace of a juvenile CA1 neuron held at $-60 \mathrm{mV}$, showing presence of slight outward shift in the picrotoxin, (G) which got intensified in the presence of SS, $(\mathbf{H})$ bar graphs show the mean outward current by PIC alone and PIC in the presence of SS. All values are mean \pm S.E.M. $(n=5)$. ${ }^{*}$ Mean with different superscripts in the same row such as $a, b,{ }^{*}$ indicates significant difference among the groups. One-way ANOVA was performed to analyze more than two experimental groups using origin 7.0. Student's $t$-test was used to examine the differences between the two experimental groups. $p<0.05$ was considered significant. 


\section{Discussion}

The effective concentration, i.e., $500 \mathrm{mg} / \mathrm{kg}$ and (or) $250 \mathrm{mg} / \mathrm{kg}$ of SS if converted to a human dose is $2430 \mathrm{mg} /$ day containing a choline concentration of $0.8312 \mathrm{mg} /$ day for an average body weight of $60 \mathrm{~kg}$. Two behavioral tests, the Morris water maze and Y-maze tests, are commonly used as experimental tools for examining learning and memory. Scopolamine is a muscarinic antagonist, that affects neurotransmission in the central nervous system and leads to amnesia in animal experimental models [29]. In this study, animals treated with SS + Scop showed a less velocity, decrease escape latency, longer time spent in hidden zones (Figure 3A-D) (spatial memory), and high spontaneous alternation percentage and increase total number of arm entries (working memory) (Figure 4A,B) than the group treated with scopolamine alone $[27,29]$. The SS improved cognitive function as well as attenuate scopolamine induced memory impairment which was clearly evident in both models of Morris water maze test and Y-maze test. These results suggest that SS could alleviate memory impairment in animal models [30].

Previous studies have shown that basal forebrain nuclei serve as major sources of cholinergic projection neurons to cortex and hippocampus [31,32]. Alteration of cholinergic neurons in the hippocampus and frontal cortex are implicated in the cognitive disorders [3]. Acetylcholine synthesized by choline acetyl transferase in neurons and hydrolyzed by acetylcholine transferase after its release, is essential to memory process [22]. Therapies designed to reverse the cholinergic deficit in large measure based on their importance in cognition. A previous study reported that increased acetylcholine in the hippocampus and frontal cortex during behavioral analysis in an animal model improved memory function following the administration of almond extract [33]. Similar to earlier report, the current study found an increase in acetylcholine content in the hippocampus and frontal cortex in the SS-supplemented groups compared with the scopolamine group. Supplementation with the SS also inhibited AChE (Figure 5C) activity, and increased CAT (Figure 5B) levels in the hippocampus and frontal cortex by modulating the cholinergic system compared to the group treated with only scopolamine. This result again demonstrated that SS protects the brain from memory impairment as observed in the behavioral experiments performed in the present study.

In recent years, investigations into the cellular and molecular pathways related to neuroprotection have been performed, providing a basis for future therapies for memory disorders. NGF plays a critical role in neuronal plasticity [6]. BDNF and its receptor TrkB are implicated in neuronal survival, morphogenesis, and synaptic plasticity $[7,34]$ and also regulates memory formation. CREB leads to the neuronal synaptic plasticity underlying learning and memory through the expression of downstream target genes, such as Bcl2 [9] and EGR1 [10]. Several studies have shown that the expression of BDNF is impaired in patients with dementia, as well as in amnesia induced animal models $[8,29,35]$. Similar to earlier reports, the current study found that the mRNA expression levels of NGF, BDNF, TrkB, CREB, and other genes such as Bcl2 and EGR1 were downregulated in the scopolamine-treated group compared to the control group. Interestingly, SS supplementation upregulated the expression level of these genes in both the hippocampus (Figure 6A-F) and frontal cortex (Figure 7A-F). Contrarily, neuronal apoptosis genes, such as Bax, lead to MOMP [10] induce the release of COX-2 into the cytoplasm [12], resulting in activation of caspase-9 and caspase-3 and leading to neuronal cell death $[11,13]$. Studies have reported that an increase in expression of caspases, COX-2, and Bax can promote neuronal degeneration and alleviate the memory loss seen in dementia patients $[13,36]$. In agreement with these previous studies, upregulation of COX-2, Bax, caspase-9, and caspase- 3 was observed in both the hippocampus (Figure 6G-J) and frontal cortex (Figure 7G-J) in the scopolamine group compared to the control group, while downregulation of COX-2, Bax, caspase-9, and caspase- 3 was seen in the SS supplemented group. Based on these results, we suggest that SS might prevent memory loss via activation of neurotrophic factors or/and by inhibition of neuronal apoptosis.

The hippocampus is a powerhouse of memory and is essential for converting short-term memory into long-term memory. Specifically, the CA1 region of the hippocampus is involved in this process [28]. In this study, bath application of SS induced reproducible and short lasting inward currents in CA1 
neurons (Figure 8C,D). The inward currents persisted in the presence of TTX, a $\mathrm{Na}^{+}$channel blocker, suggesting that SS acts on CA1 neuronal membranes or dendrites directly, rather than by action potential-mediated mechanisms (Figure $8 \mathrm{E}, \mathrm{F}$ ) $[28,37]$. In addition, the inward currents induced by SS were partially blocked by picrotoxin, a broad $\mathrm{GABA}_{\mathrm{A}}$ receptor antagonist, suggesting that $\mathrm{SS}$ exhibits GABA-mimetic activity via synaptic and extrasynaptic $\mathrm{GABA}_{\mathrm{A}}$ receptors (Figure $8 \mathrm{G}, \mathrm{H}$ ). This provides evidence that SS acts directly on the GABA binding site of CA1 pyramidal neurons in the hippocampus [17]. These results are in agreement with a previous study [38], which reported that Withania somnifera acts at a GABA receptor by inhibiting $\mathrm{GABA}_{\mathrm{A}}$ receptor agonists. Another study suggested that GABAergic neurons can critically modulate the electrical activity of the hippocampus during the multiple consolidation process of memory storage [39]. This result provides clear evidence that $S S$ has a $\mathrm{GABA}_{\mathrm{A}}$ receptor activation effect and may play an important role in neuroprotection and neurite outgrowth via regulation and activation of $\mathrm{GABA}_{\mathrm{A}}$ receptors.

In summary, dementia has been reported to be associated with the down regulation of levels of NGF-BDNF-CREB, signaling [30] which in turn it increases the mRNA expression level of apoptotic genes [36] which leads to cholinergic dysfunction and memory deficit [33]. Taken together, the results of this study demonstrated that SS can prevent and protect against scopolamine-induced memory impairment by regulating the NGF-BDNF-CREB signaling pathway (Figure 9) and inhibiting AChE expression. This study also revealed that $\mathrm{SS}$ acts through $\mathrm{GABA}_{\mathrm{A}}$ receptors in $\mathrm{CA} 1$ pyramidal neurons of the hippocampus. These data indicate that a daily intake of SS extract via dietary supplementation may produce memory-enhancing effects and prevent dementia in humans. However, further studies are needed to explore the direct mechanism of SS action, along with its clinical safety using consecutive clinical trial.

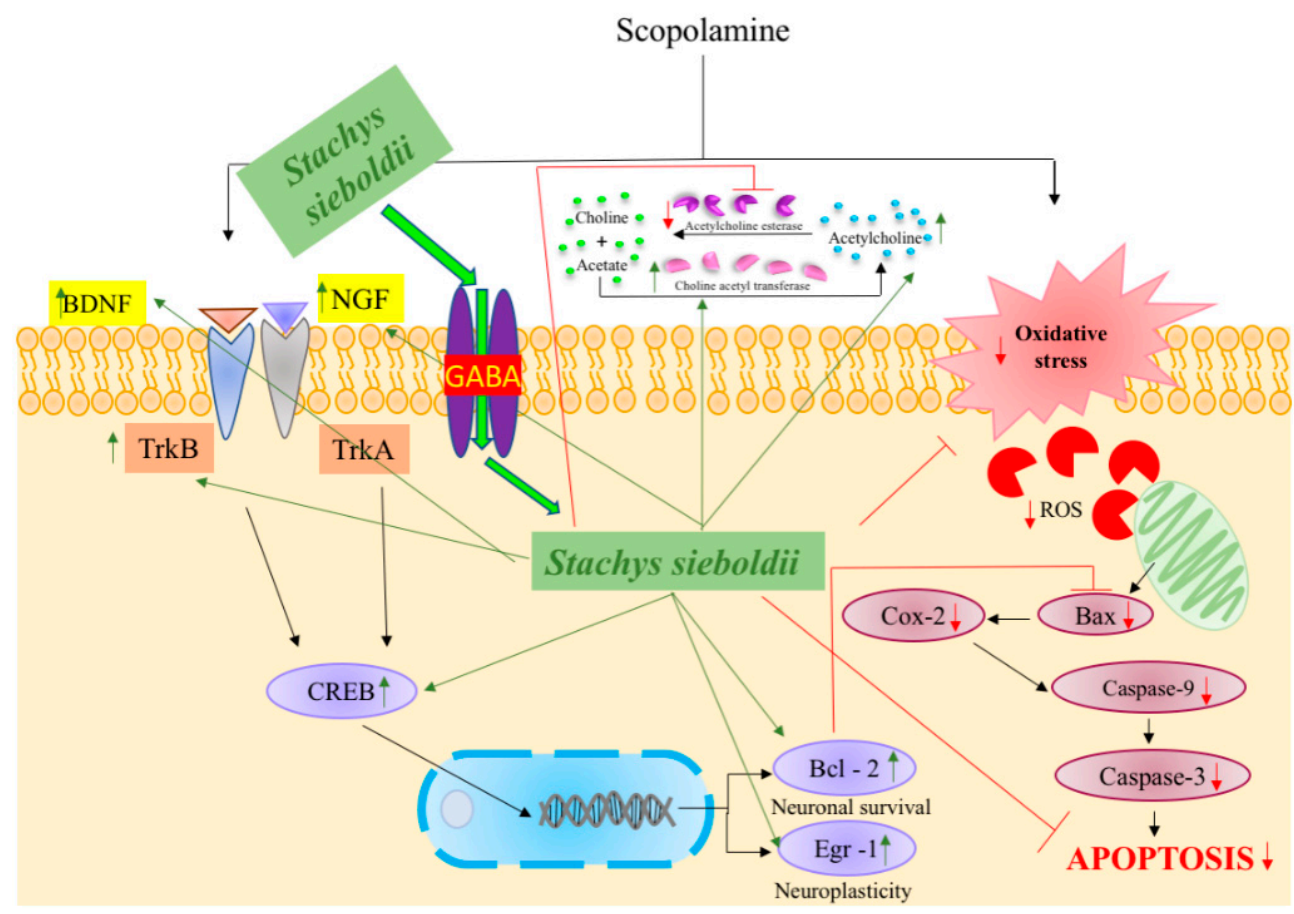

Figure 9. Mechanisms underlying the effects of Stachys sieboldii (SS). SS downregulated cellular apoptosis signaling cascade such as Bax, COX-2, Caspase-9 and Caspase-3, which results in the upregulation of memory enhancement-related gene such as NGF, BDNF, TrkB, and CREB, and its downstream targets Bcl-2 and Egr-1 which are involved in neuroplasticity and neuronal survival in the cerebral cortex and hippocampus. SS also reduced the AChE and increased the CAT and ACh in brain. Thus, SS prevents memory impairments through the NGF-CREB-BDNF signaling pathway and inhibitory property of AChE. 
Supplementary Materials: The following are available online at http:/ / www.mdpi.com/2072-6643/10/7/917/s1, Figure S1: UHPLC-MS/MS of crude extract of Stachys Sieboldii; Table S1: Compounds present in crude extract of Stachys Sieboldii.

Author Contributions: Y.S.C., M.K. and V.A.R. conceived and designed the study. V.A.R. conducted experiments and analyzed the data from in vitro and in vivo studies. S.K.H. and V.A.R. designed, performed, and analyzed the electrophysiological studies. V.A.R. wrote the manuscript. All authors reviewed the manuscript and approved the final version.

Acknowledgments: This research was financially supported by the Ministry of Trade, Industry, and Energy (MOTIE) of Korea, under the "Regional Specialized Industry Development Program" supervised by the Korea Institute for Advancement of Technology (KIAT).

Conflicts of Interest: The authors declare no conflict of interest.

\section{Abbreviations}

SS, Stachys sieboldii; WHO, World Health Organization; ACh, Acetylcholine; CAT, Cholineacetyl transferase; AChE, Acetylcholineesterase; NGF, Nerve growth factor; BDNF, Brain-derived neurotrophic factor; TrkB, Tropomyosin receptor kinase B; LTM, long term memory; CREB, cAMP response element binding protein; Bcl2, B-cell lymphoma 2; EGR1, early growth response 1; MOMP, Mitochondrial outer membrane permeabilization; COX-2, cyclooxygenase-2 Bax, Bcl2 associated X protein; GABA, $\gamma$-aminobutyric acid; TTX, tetrodotoxin; PIC, Picrotoxin.

\section{References}

1. Canevelli, M.; Blasimme, A.; Cesari, M. Societal and global implications of the "dementia epidemic": The example of the London Heathrow airport. Eur. J. Epidemiol. 2017, 32, 347-348. [CrossRef] [PubMed]

2. Tarawneh, R.; Holtzman, D.M. The clinical problem of symptomatic Alzheimer disease and mild cognitive impairment. Cold Spring Harb. Perspect. Med. 2012, 2, a006148. [CrossRef] [PubMed]

3. Davies, P.; Maloney, A.J.F. Selective loss of central cholinergic neurons in Alzheimer's disease. Lancet 1976, 2, 1403. [CrossRef]

4. García-Ayllón, M.S.; Riba-Llena, I.; Serra-Basante, C.; Alom, J.; Boopathy, R.; Sáez-Valero, J. Altered Levels of Acetylcholinesterase in Alzheimer Plasma. PLoS ONE 2010, 5, e8701. [CrossRef] [PubMed]

5. Mufson, E.J.; Counts, S.E.; Perez, S.E.; Ginsberg, S.D. Cholinergic system during the progression of Alzheimer's disease: Therapeutic implications. Expert Rev. Neurother. 2008, 8, 703-1718. [CrossRef] [PubMed]

6. Conner, J.M.; Franks, K.M.; Titterness, A.K.; Russell, K.; Merrill, D.A.; Christie, B.R.; Sejnowski, T.J.; Tuszynski, M.H. NGF is essential for hippocampal plasticity and learning. J. Neurosci. 2009, 29, 10883-10889. [CrossRef] [PubMed]

7. Yamada, K.; Nabeshima, T. Brain-derived neurotrophic factor/TrkB signaling in memory processes. J. Pharmacol. Sci. 2003, 91, 267-270. [CrossRef] [PubMed]

8. Alonso, M.; Bekinschtein, P.; Cammarota, M.; Vianna, M.R.; Izquierdo, I.; Medina, J.H. Endogenous BDNF is required for long-term memory formation in the rat parietal cortex. Learn. Mem. 2005, 12, 504-510. [CrossRef] [PubMed]

9. Li, Q.; Zhao, H.F.; Zhang, Z.F.; Liu, Z.G.; Pei, X.R.; Wang, J.B.; Li, Y. Long-term administration of green tea catechins prevents age-related spatial learning and memory decline in C57BL/6 J mice by regulating hippocampal cyclic amp-response element binding protein signaling cascade. Neuroscience 2009, 159, 1208-1215. [CrossRef] [PubMed]

10. Maddox, S.A.; Monsey, M.S.; Schafe, G.E. Early growth response gene 1 (Egr-1) is required for new and reactivated fear memories in the lateral amygdala. Learn. Mem. 2011, 18, 24-38. [CrossRef] [PubMed]

11. D'Amelio, M.; Cavallucci, V.; Cecconi, F. Neuronal caspase-3 signaling: Not only cell death. Cell Death Differ. 2010, 17, 1104-1114. [CrossRef] [PubMed]

12. Shaw, K.N.; Commins, S.; O'mara, S.M. Deficits in spatial learning and synaptic plasticity induced by the rapid and competitive broad-spectrum cyclooxygenase inhibitor ibuprofen are reversed by increasing endogenous brain-derived neurotrophic factor. Eur. J. Neurosci. 2003, 17, 2438-2446. [CrossRef] [PubMed]

13. Li, Z.; Sheng, M. Caspases in synaptic plasticity. Mol. Brain 2012, 5, 15. [CrossRef] [PubMed]

14. Brady, M.L.; Pilli, J.; Lorenz-Guertin, J.M.; Das, S.; Moon, C.E.; Graff, N.; Jacob, T.C. Depolarizing, inhibitory GABA type A receptor activity regulates GABAergic synapse plasticity via ERK and BDNF signaling. Neuropharmacology 2018, 128, 324-339. [CrossRef] [PubMed] 
15. Gonzalez, M.I. Brain-derived neurotrophic factor promotes gephyrin protein expression and GABAA receptor clustering in immature cultured hippocampal cells. Neurochem. Int. 2014, 72, 14-21. [CrossRef] [PubMed]

16. Johnston, G.A.; Hanrahan, J.R.; Chebib, M.; Duke, R.K.; Mewett, K.N. Modulation of ionotropic GABA receptors by natural products of plant origin. Adv. Pharmacol. 2006, 54, 285-316. [PubMed]

17. Bhattarai, J.P.; Park, S.J.; Han, S.K. Potentiation of NMDA receptors by Withania somnifera on hippocampal CA1 pyramidal neurons. Am. J. Chin. Med. 2013, 41, 503-513. [CrossRef] [PubMed]

18. Vijaya Abinaya, R.; Pichiah, P.B.T.; Sara Thomas, S.; Kim, S.G.; Han, D.W.; Song, Y.S.; Oh, S.H.; Cha, Y.S. $\gamma$-amino butyric acid-enriched barley bran lowers adrenocorticotropic hormone and corticosterone levels in immobilized stressed rats. J. Food Biochem. 2017, 41, e12324. [CrossRef]

19. Yamahara, J.; Kitani, T.; Kobayashi, H.; Kawahara, Y. Studies on Stachys sieboldii MIQ. II. Anti-anoxia action and the active constituents. Yakugaku zasshi: J. Pharm. Soc. Jpn. 1990, 110, 932-935. [CrossRef]

20. Hayashi, K.; Nagamatsu, T.; Ito, M.; Hattori, T.; Suzuki, Y. Acteoside, a component of Stachys sieboldii MIQ, may be a promising antinephritic agent: Effect of acteoside on crescentic-type anti-GBM nephritis in rats. Jpn. J. Pharmacol. 1994, 65, 143-151. [CrossRef] [PubMed]

21. Yin, J.; Yang, G.; Wang, S.; Chen, Y. Purification and determination of stachyose in Chinese artichoke (Stachys Sieboldii Miq.) by high-performance liquid chromatography with evaporative light scattering detection. Talanta 2006, 70, 1208-1212. [CrossRef] [PubMed]

22. Vijaya Abinaya, R.; Kim, M.; Lee, S.J.; Cha, Y.S. Protective effects of Stachys sieboldii MIQ extract in SK-N-SH cells and its memory ameliorative effect in mice. J. Food Biochem. 2017, 41, e12411. [CrossRef]

23. Nishimura, H.; Sasaki, H.; Inagaki, N.; Masao, C.; Chen, Z.; Mitsuhashi, H. Nine phenethyl alcohol glycosides from Stachys sieboldii. Phytochemistry 1991, 30, 965-969. [CrossRef]

24. Agrawal, R.; Tyagi, E.; Shukla, R.; Nath, C. Effect of insulin and melatonin on acetylcholinesterase activity in the brain of amnesic mice. Behav. Brain Res. 2008, 189, 381-386. [CrossRef] [PubMed]

25. Freireich, E.J.; Gehan, E.A.; Rall, D.P.; Schmidt, L.H.; Skipper, H.E. Quantitative comparison of toxicity of anticancer agents in mouse, rat, hamster, dog, monkey and man. Cancer Chemother Rep. 1966, 50, $219-244$. [PubMed]

26. Morris, R. Developments of a water-maze procedure for studying spatial learning in the rat. J. Neurosci. Methods 1984, 11, 47-60. [CrossRef]

27. Ma, M.; Chen, Y.; He, J.; Zeng, T.; Wang, J. Effects of morphine and its withdrawal on Y-maze spatial recognition memory in mice. Neuroscience 2007, 147, 1059-1065. [CrossRef] [PubMed]

28. Bhattarai, J.P.; Han, S.K. Phasic and tonic type A $\gamma$-Aminobutryic acid receptor mediated effect of Withania somnifera on mice hippocampal CA1 pyramidal Neurons. J. Ayurveda Integr. Med. 2014, 5, $216-222$. [PubMed]

29. Sattayasai, J.; Chaonapan, P.; Arkaravichie, T.; Soi-Ampornkul, R.; Junnu, S.; Charoensilp, P.; Samer, J.; Jantaravinid, J.; Masaratana, P.; Suktitipat, B.; et al. Protective effects of mangosteen extract on $\mathrm{H}_{2} \mathrm{O}_{2}$-induced cytotoxicity in SK-N-SH cells and scopolamine-induced memory impairment in mice. PLoS ONE 2013,8, e85053. [CrossRef] [PubMed]

30. Um, M.Y.; Lim, D.W.; Son, H.J.; Cho, S.; Lee, C. Phlorotannin-rich fraction from Ishige foliacea brown seaweed prevents the scopolamine-induced memory impairment via regulation of ERK-CREB-BDNF pathway. J. Funct. Foods 2018, 40, 110-116. [CrossRef]

31. Ballinger, E.; Ananth, M.; Talmage, D.A.; Role, L. Basal Forebrain Cholinergic Circuits and Signaling in Cognition and Cognitive Decline. Neuron 2016, 91, 1199-1218. [CrossRef] [PubMed]

32. Mesulam, M.M.; Mufson, E.J.; Levey, A.I.; Wainer, B.H. Cholinergic innervation of cortex by the basal forebrain: Cytochemistry and cortical connections of the septal area, diagonal band nuclei, nucleus basalis (substantia innominata), and hypothalamus in the rhesus monkey. J. Comp. Neurol. 1983, 214, 170-197. [CrossRef] [PubMed]

33. Batool, Z.; Sadir, S.; Liaquat, L.; Tabassum, S.; Madiha, S.; Rafiq, S.; Haider, S. Repeated administration of almonds increases brain acetylcholine levels and enhances memory function in healthy rats while attenuates memory deficits in animal model of amnesia. Brain Res. Bull. 2016, 120, 63-74. [CrossRef] [PubMed]

34. Reichardt, L.F. Neurotrophin-regulated signalling pathways. Philos. Trans. R. Soc. Lond. B. Biol. Sci. 2006, 361, 1545-1564. [CrossRef] [PubMed] 
35. Borba, E.M.; Duarte, J.A.; Bristot, G.; Scotton, E.; Camozzato, A.L.; Chaves, M.L.F. Brain-derived neurotrophic factor serum levels and hippocampal volume in mild cognitive impairment and dementia due to alzheimer disease. Dement. Geriatr. Cogn. Disord. Extra 2016, 6, 559-567. [CrossRef] [PubMed]

36. Friedlander, R.M. Apoptosis and caspases in neurodegenerative diseases. N. Engl. J. Med. 2003, 348, 1365-1375. [CrossRef] [PubMed]

37. Bhattarai, J.P.; Ah Park, S.; Han, S.K. The methanolic extract of Withania somnifera ACTS on GABAA receptors in gonadotropin releasing hormone (GnRH) neurons in mice. Phytother. Res. 2010, 24, 1147-1150. [PubMed]

38. Mehta, A.; Binkley, P.; Gandhi, S.; Ticku, M. Pharmacological effects of Withania somnifera root extract on GABAA receptor complex. Indian J. Med. Res. 1991, 94, 312-315. [PubMed]

39. Porcher, C.; Hatchett, C.; Longbottom, R.E.; McAinch, K.; Sihra, T.S.; Moss, S.J.; Thomson, A.M.; Jovanovic, J.N. Positive feedback regulation between $\gamma$-aminobutyric acid type A (GABAA) receptor signaling and brain-derived neurotrophic factor (BDNF) release in developing neurons. J. Biol. Chem. 2011, 286, 21667-21677. [CrossRef] [PubMed]

(C) 2018 by the authors. Licensee MDPI, Basel, Switzerland. This article is an open access article distributed under the terms and conditions of the Creative Commons Attribution (CC BY) license (http:/ / creativecommons.org/licenses/by/4.0/). 